Article

\title{
Integrated Proteomics and Metabolomics Analysis of Perirenal Adipose Tissue in Obese Rabbits Treated with a Restricted Diet
}

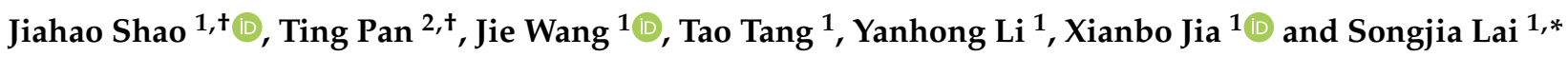 \\ 1 College of Animal Science and Technology, Sichuan Agricultural University, Chengdu 611130, China; \\ shaojh1997@126.com (J.S.); wjie68@163.com (J.W.); m18483220592@163.com (T.T.); \\ lyh81236718@163.com (Y.L.); jaxb369@sicau.edu.cn (X.J.) \\ 2 College of Veterinary Medicine, Sichuan Agricultural University, Chengdu 611130, China; \\ panting555666@163.com \\ * Correspondence: laisj5794@163.com \\ + These authors contributed equally to this work.
}

Citation: Shao, J.; Pan, T.; Wang, J.;

Tang, T.; Li, Y.; Jia, X.; Lai, S.

Integrated Proteomics and

Metabolomics Analysis of Perirenal Adipose Tissue in Obese Rabbits

Treated with a Restricted Diet. Biology 2021, 10, 321. https://doi.org/

10.3390/biology10040321

Academic Editor: Ansgar Poetsch

Received: 25 February 2021

Accepted: 8 April 2021

Published: 12 April 2021

Publisher's Note: MDPI stays neutral with regard to jurisdictional claims in published maps and institutional affiliations.

Copyright: (c) 2021 by the authors. Licensee MDPI, Basel, Switzerland. This article is an open access article distributed under the terms and conditions of the Creative Commons Attribution (CC BY) license (https:/ / creativecommons.org/licenses/by/ $4.0 /)$.
Simple Summary: Nowadays, obesity and obesity-related diseases are rapidly increasing in most countries and regions. In this context, about $25-50 \%$ of people show that they are trying to lose weight and this trend is especially prominent among women. Here, obese rabbits were used as a model to study the effects of dieting on the molecular change of perirenal adipose tissue by integrating proteomics and metabolomics analysis. Our results indicate that 343 proteins and 150 metabolites were markedly changed and these molecules are associated with amino acid metabolism, lipid metabolism, and membrane and cytoskeleton reconstruction. Interestingly, some inflammation-related molecules such as mevalonic acid, arachidonic acid, 15(S)-HpETE, cholecalciferol, hydrocortisone, lipoxin B4, lithocholic acid, etc. were differently changed and these molecules may be the key roles to fight inflammation induced by a high-fat diet. In conclusion, this study provides a comprehensive overview of the molecular profile of dieting-mediated weight loss and may provide some help for the prevention and treatment of obesity.

Abstract: In recent years, many people have shown an excess of fat accumulation. Known as obesity, this lesion poses an increased risk for multiple diseases, such as endocrine disease, diabetes, and cancer, and has reached epidemic proportions. Accompanied by the development of obesity, concern over body image and weight loss behavior is a growing social problem and public health threat, causing concern for many health professionals. However, the consequences of rapid weight loss remain largely unclear. Here, we applied an integrated proteomics and metabolomics analysis to investigate the effects of dieting on the proteins and metabolites in obese rabbits. Our study revealed that 343 differentially expressed proteins (136 upregulated and 207 downregulated) and 150 differentially expressed metabolites (91 upregulated and 59 downregulated) were identified. These molecules are mainly involved in the biological processes, including amino acid metabolism, lipid metabolism, and membrane and cytoskeleton reconstruction. The integrated analysis found that mevalonic acid, arachidonic acid, 15(S)-HpETE, cholecalciferol, hydrocortisone, lipoxin B4, lithocholic acid, etc. were associated with multiple pathways, and they may be the key factors to fight inflammation induced by a high-fat diet (HFD). Overall, this study provides further insight into the consequences of dieting-mediated weight loss and may contribute to the prevention and treatment of obesity.

Keywords: proteomics; metabolomics; rabbits; dieting

\section{Introduction}

Nowadays, obesity promotes an increased risk for a series of diseases, including endocrine disease, diabetes, cancer, etc., and its incidence rapidly increases in most countries and even in some lower-income countries [1]. Obesity is visualized as an excess of adipose 
tissue, which is an endocrine organ and energy storage organ and plays a key role in maintaining physiological activities [2]. Obesity is a complex pathological process, and available and cheaper highly palatable and fat-dense foods are important contributors $[3,4]$. In this context, young females are suffering from a higher rate of obesity risk than males [5]. Meanwhile, concern over body image and weight loss behavior is particularly common in adolescent females [6]. Overall, slimness is often considered the norm for physical appearance. Dieting seems to be the first choice for obese people to lose weight because of its easy applicability. Despite that, there are some controversies about dieting to lose weight, such as the measurement of dieting and psychological reactions for different people $[7,8]$. The harmful side effects of the thinness pursuit are a growing social problem and public health threat, causing concern for many health professionals, and the consequences of dieting need to be taken seriously [9].

Omics technology is developing at a high rate of speed and is broadly applied as a powerful tool for various studies, such as the examination of pathophysiological processes, identification of biomarkers, molecular profiling, and characterization of complex biochemical systems [10]. The physiological activity is complex, as using a single omics makes it hard to fully reveal the potential mechanism. Thus, to identify the potential biological molecules and improve the understanding of the overall biological mechanisms, an integrated analysis of multiple omics is necessary. Moreover, different omics are complementary to each other and contribute to increasing the reliability of the results. Among these integrated omics, an integrated proteomics and metabolomics analysis is a useful combination to reveal the mechanisms of molecular regulation on phenotypic changes. To our knowledge, no combination of proteomics and metabolomics has been performed to analyze the molecular changes of perirenal adipose tissue from obese people or animals treated with dieting. Thus, we aimed to gain further understanding of the molecular mechanisms of losing weight through an integrated proteomics and metabolomics analysis from the obese rabbit model under dietary restriction treatment.

\section{Materials and Methods}

\subsection{Animals}

The animals were sixteen female Tianfu black rabbits (a new variety screened by Sichuan Agricultural University) that were 35 days old at the initiation of this trial. Rabbits were kept in individual cages $(600 \mathrm{~mm} \times 600 \mathrm{~mm} \times 500 \mathrm{~mm})$ and were placed indoors ( 21 to $23{ }^{\circ} \mathrm{C}, 60 \%$ humidity). Moreover, rabbits were treated with a two-stage diet plan. In the first stage, rabbits were fed a high-fat diet (HFD; a 10\% lard was added to the standard diet, $120 \mathrm{~g} / \mathrm{d}$ ) for 35 days. In the second stage, HFD rabbits were treated with a restricted diet (RD; standard diet, $20 \mathrm{~g} / \mathrm{d}$ ) for 20 days. Before each stage, rabbits were housed undisturbed for 7 days to adapt to the change. Animals were classified as obese using the method described in our previous study [11]. Briefly, body weight, body length, bust length, and adipose tissue weight were used as the markers of obesity. Lastly, unqualified (weak, disabled, and sick) rabbits were eliminated, and six rabbits from the HFD (77 days) and RD (104 days) groups were selected for sampling. All experimental protocols were performed under the direction of the Institutional Animal Care and Use Committee from the College of Animal Science and Technology, Sichuan Agricultural University, China (DKY-B2019202015).

\subsection{Histomorphological Observation}

To examine the histomorphological changes of perirenal adipose tissue, selective rabbits were sacrificed (electric shock and then exsanguination) for sampling, and perirenal adipose tissues were stained with hematoxylin-eosin (HE). Briefly, tissue samples were fixed with a $10 \%$ neutral formaldehyde fixator for $24 \mathrm{~h}$ and then washed with water. The tissue specimens were successively dehydrated and embedded in paraffin, followed by HE staining. Finally, about 5- $\mu \mathrm{m}$-thick sections were obtained using a microtome (RM2235, Leica, Nussloch, Germany). Moreover, an optical microscope (DM1000, Leica, Nussloch, 
Germany) was used to capture images at a $200 \times$ field of view, and the relevant parameters (adipocytes area, diameter, number, and density) were measured by image-pro plus 6.0 software (Media Cybernetics, Inc, Rockville, MD, USA).

\subsection{TMT Labeling}

Total protein was extracted and measured using a commercial kit (Sangon, Shanghai, China) and the BCA Protein Assay Kit (Beyotime, Jiangsu, China) following the manufacturer's protocols, respectively. Only qualified protein (concentration $>1 \mathrm{mg} / \mathrm{mL}$ ) was used for further trial. One hundred and twenty micrograms of protein were diluted with a dissolution buffer (1.5- $\mu \mathrm{g}$ trypsin and $500 \mu \mathrm{L}$ of $100-\mathrm{mM}$ triethylammonium bicarbonate (TEAB) buffer to $100 \mu \mathrm{L}$ ) and digested at $37^{\circ} \mathrm{C}$. Subsequently, 1.5- $\mu \mathrm{g}$ trypsin and $\mathrm{CaCl}_{2}$ were added to the sample. The next day, formic acid was mixed with the above-digested sample (adjusted $\mathrm{pH}$ under 3), and the mixture was centrifuged at $12,000 \times \mathrm{g}$ for $5 \mathrm{~min}$ at an indoor temperature. The supernatant was loaded onto an C18 desalting column (Phenomenex, Torrance, CA, USA), washed with washing buffer ( $0.1 \%$ formic acid and $3 \%$ acetonitrile) 3 times, then eluted by elution buffer ( $0.1 \%$ formic acid and $70 \%$ acetonitrile). The eluent was collected and lyophilized. One hundred microliters of 0.1-M TEAB buffer and $41 \mu \mathrm{L}$ of acetonitrile-dissolved tandem mass tag (TMT) reagent were added and incubated for $2 \mathrm{~h}$. The mixture was stopped by adding $8 \%$ ammonia. Finally, the labeling sample was desalted, pooled, and lyophilized.

\subsection{Separation of Fractions}

Mobile phases A ( $2 \%$ acetonitrile) and B ( $98 \%$ acetonitrile) were used to develop a gradient elution. The lyophilized powder was dissolved in solution A and centrifuged at $12,000 \times g$ for $10 \mathrm{~min}$ at an indoor temperature. The sample was fractionated using a C18 column (4.6-mm ID, 250-mm length, and 5- $\mu \mathrm{m}$ particles) on a Rigol L3000 high performance liquid chromatography (HPLC) system, and the column oven was set as $50{ }^{\circ} \mathrm{C}$. The eluates were monitored at UV $214 \mathrm{~nm}$, collected for a tube per minute, and combined into 10 fractions. All fractions were dried under vacuum and then reconstituted in $0.1 \%(v / v)$ formic acid $(\mathrm{FA})$ in water.

\subsection{LC-MS/MS Analysis}

The tryptic peptides were detached by using the EASY-nLC 1200 ultra performance liquid chromatography (UHPLC) system (Thermo Fisher Scientific, Waltham, MA, USA). Subsequently, the separated peptides were analyzed by using a Q Exactive HF-X mass spectrometer (Thermo Fisher Scientific, Waltham, MA, USA), with an ion source of Nanospray Flex ${ }^{\mathrm{TM}}$ electron spray lonization (ESI). The ion source was set at $2.3 \mathrm{kV}$, and the capillary temperature of the ion transport was $320^{\circ} \mathrm{C}$. The $\mathrm{m} / \mathrm{z}$ scan range was 350 to 1500 , and the resolution was 60,000 . The automatic gain control target value was $3 \times 10^{6}$, and the maximum ion injection time was $20 \mathrm{~ms}$. The top 40 precursors with the highest contents in the full scans were selected and fragmented by higher-energy collisional dissociation and analyzed by tandem mass spectrometry (MS/MS) (resolution: 45,000, automatic gain control (AGC) target value: $5 \times 10^{4}$, maximum ion injection time: $86 \mathrm{~ms}$, normalized collision energy: $32 \%$, intensity threshold: $1.2 \times 10^{5}$, and dynamic exclusion parameter: $20 \mathrm{~s}$ ).

\subsection{Proteomics Data Processing and Analysis}

The Proteome Discoverer 2.2 (PD; Thermo) was used to obtain the MS/MS results. The parameters were set as follows: (a) mass tolerance for a precursor ion was $10 \mathrm{ppm}$, and the mass tolerance for fragment ion was $0.02 \mathrm{Da}$, (b) carbamidomethyl was specified as a fixed modification, but the oxidation of methionine and TMT plex were specified as dynamic modifications, (c) acetylation and TMT plex were specified as N-terminal modification in PD 2.2, and (d) a maximum of 2 miscleavage sites were allowed. The protein concluding at least one unique peptide and meeting criteria (false discovery rate (FDR) $\leq 1 \%$ ) was retained for further trial. The proteins with $p<0.05$ and fold changes $\geq 1.2$ or fold changes 
$\leq 0.83$ were identified as upregulated or downregulated differentially expressed (DE) protein, respectively. Gene Ontology (GO; http:/ /www.geneontology.org/, accessed on 16 May 2020) was used to analyze the functional annotation.

\subsection{UHPLC-MS/MS Analysis}

The detailed procedure for sample preparation was described previously [12]. Then, the metabolites were analyzed using a UHPLC system (Thermo Fisher Scientific, Waltham, MA, USA) coupled with an Orbitrap Q Exactive ${ }^{\mathrm{TM}}$ HF-X mass spectrometer (Thermo Fisher Scientific, Waltham, MA, USA) by Novogene Co., Ltd. (Beijing, China). Briefly, the sample was injected into a column (2.1-mm ID, 100-mm length, and 1.9- $\mu \mathrm{m}$ particles) using a linear gradient at a flow rate of $0.2 \mathrm{~mL} / \mathrm{min}$. The eluents for the positive polarity mode were eluent $\mathrm{A}(0.1 \% \mathrm{FA}$ in water) and eluent $\mathrm{B}$ (methanol) and, for the negative polarity mode, were eluent A (5-mM ammonium acetate) and eluent B (methanol). The searched parameters are set as follows: (a) spray voltage was $3.2 \mathrm{kV}$, (b) capillary temperature was $320^{\circ} \mathrm{C}$, (c) sheath gas flow was $40 \mathrm{arb}$, and aux gas flow was 10 arb.

\subsection{Metabolomics Data Processing and Analysis}

Data generated by UHPLC-MS/MS were loaded into Compound Discoverer 3.1 (CD; Thermo) to process the peak alignment, peak picking, and quantitation. Peak intensities were normalized to the total spectral intensity. The normalized data were used for a multivariate statistical analysis and were matched with the mzCloud (https: //www.mzcloud.org/, accessed on 16 May 2020), mzVault, and MassList databases to obtain the accurate result. Normal transformations were attempted using the area normalization method when the data were not normally distributed. To better observe the inter-group distributions and otherness, SIMCA- $p 11.0$ software was used to carry out a partial least squares discriminant analysis (PLS-DA). Metabolites were considered to be differentially expressed, with a threshold variable importance in the projection (VIP) $>1$ and fold changes $>1.5$ or fold changes $<0.667$ and $p<0.05$. All DE metabolites were annotated with the Human Metabolome database (HMDB; https://hmdb.ca/metabolites, accessed on 16 May 2020) and LIPID MAPS database (https://www.lipidmaps.org/, accessed on 16 May 2020) to obtain a systematized overview of these DE metabolites. Moreover, the metabolite-metabolite interaction network helps to highlight potential functional relationships between a wide set of annotated metabolites and was built using the MetaboAnalyst database (https://www.metaboanalyst.ca/MetaboAnalyst/home. xhtml, accessed on 16 May 2020).

\subsection{Integrated Analysis of Proteomics and Metabolomics}

To further analyze the significantly changed pathways in both proteomics and metabolomics, we integrated the analysis of the DE proteins and DE metabolites in the Integrated Molecular Pathway Level Analysis (IMPaLA) database (http:/ /impala. molgen.mpg.de/, accessed on 16 May 2020). The IMPaLA database combines information from multiple databases, such as Reactome (http:/ / www.reactome.org, accessed on 16 May 2020), Wikipathways (https:/ / www.wikipathways.org/index.php/WikiPathways, accessed on 16 May 2020), Kyoto Encyclopedia of Eenes and Eenomes (KEGG; https: / / www.kegg.jp/, accessed on 16 May 2020), and HumanCyc (https:/ / humancyc.org/, accessed on 16 May 2020) databases.

\subsection{Statistical Analysis}

The data are expressed as the mean \pm SEM. Statistical analyses were performed using the software SPSS 22.0 (Chicago, IL, USA). Volcano map and a hierarchical clustering analysis were performed using the $\mathrm{R}$ package. Moreover, we applied a $t$-test to calculate the statistical significance, and $p<0.05$ was considered as statistically significant. 


\section{Results}

\subsection{Histomorphological Changes in the Perirenal Adipose Tissue of Dieting Rabbits}

We performed HE staining on perirenal adipose tissue from the HFD rabbits and RD rabbits to measure the histomorphological changes (Figure 1). The detailed parameters (adipocytes area, diameter, number, and density) are shown in Table 1. When comparing the significant analysis data between the HFD and RD rabbits, we found that the adipocytes area $(p<0.05)$ and adipocytes diameter $(p<0.01)$ were significantly lower in the RD rabbits, but the adipocytes number $(p<0.01)$ and adipocytes density $(p<0.01)$ in the RD rabbits were significantly higher than in the HFD rabbits. These observations indicate that dieting caused adipocytes to appear smaller in the RD group.
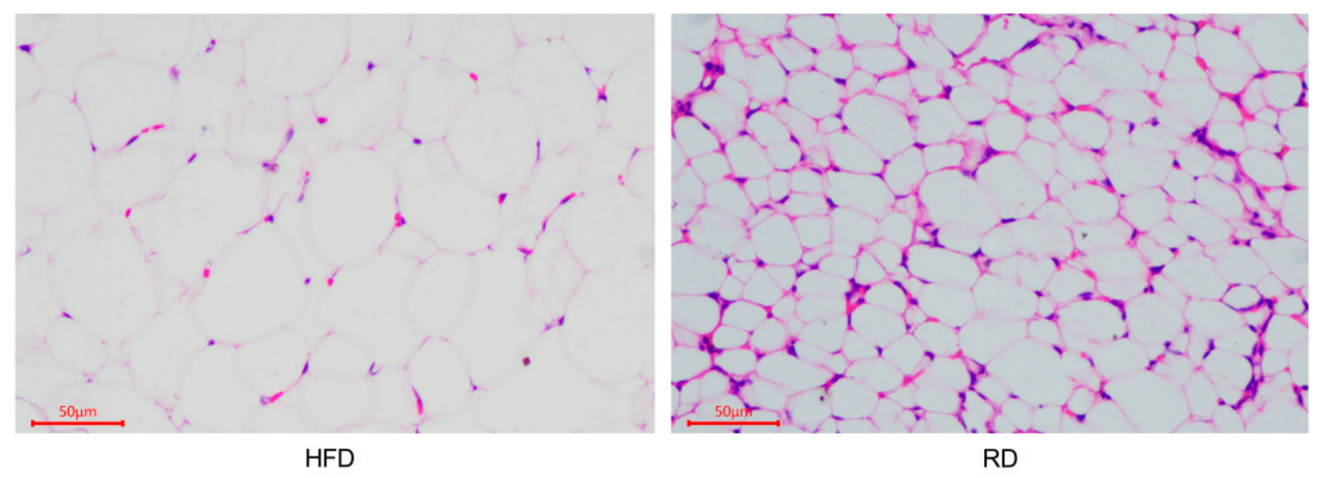

Figure 1. Histomorphological changes in the perirenal adipose tissue of dieting rabbits. Histomorphological image of the perirenal adipose tissue from the high-fat diet (HFD) and restricted diet (RD) rabbits.

Table 1. Adipocytes area, diameter, number, and density in high-fat diet (HFD) and restricted diet (RD) rabbits.

\begin{tabular}{ccc}
\hline Parameters & HFD & RD \\
\hline Adipocytes area $\left(\mu \mathrm{m}^{2}\right)$ & $1098 \pm 92.13$ & $793.6 \pm 57.55^{*}$ \\
Adipocytes diameter $(\mu \mathrm{m})$ & $39.81 \pm 1.485$ & $34.44 \pm 1.038^{* *}$ \\
Adipocytes number & $37.22 \pm 4.651$ & $54.89 \pm 3.3448^{* *}$ \\
Adipocytes density $\left(\right.$ number $\left./ \mathrm{mm}^{2}\right)$ & $417.8 \pm 52.32$ & $619.4 \pm 37.73^{* *}$
\end{tabular}

Data are shown as the mean \pm SEM $(n=9)$. Three HFD and three RD rabbits were used for sampling, and three duplicate samples were collected from each rabbit. A $t$-test was used for the differences between the HFD and RD rabbits. ${ }^{*} p<0.05$ and ${ }^{* *} p<0.01$.

\subsection{Identification and Analysis of DE Proteins}

A total of 4922 proteins (at least one peptide and FDR $\leq 1 \%$ ) were identified between the HFD and RD groups (Table S1). According to the selection criteria, of the 4922 investigated proteins, 343 proteins were classed as DE proteins in the perirenal adipose tissue between the HFD and RD groups (Table S2). Among these, 136 proteins were upregulated and 207 proteins were downregulated in the RD group compared with the HFD group (Figure 2a). DE proteins with similar expression levels across different samples were screened and grouped using a hierarchical cluster analysis (Figure 2b).

To further determine and classify the DE proteins according to their biological functions, we performed a Gene Ontology (GO) analysis with $343 \mathrm{DE}$ proteins and GO terms related to DE proteins. The analysis results revealed that $342 \mathrm{GO}$ terms were annotated, and $18 \mathrm{GO}$ terms in biological processes (BP), 4 GO terms in cellular components (CC), and $22 \mathrm{GO}$ terms in molecular functions (MF) were significantly enriched (Table S3 and Figure 2c). Among these, some GO terms were involved in the lipase activity, actin binding, phosphoric diester hydrolase activity, and actin filament binding. 


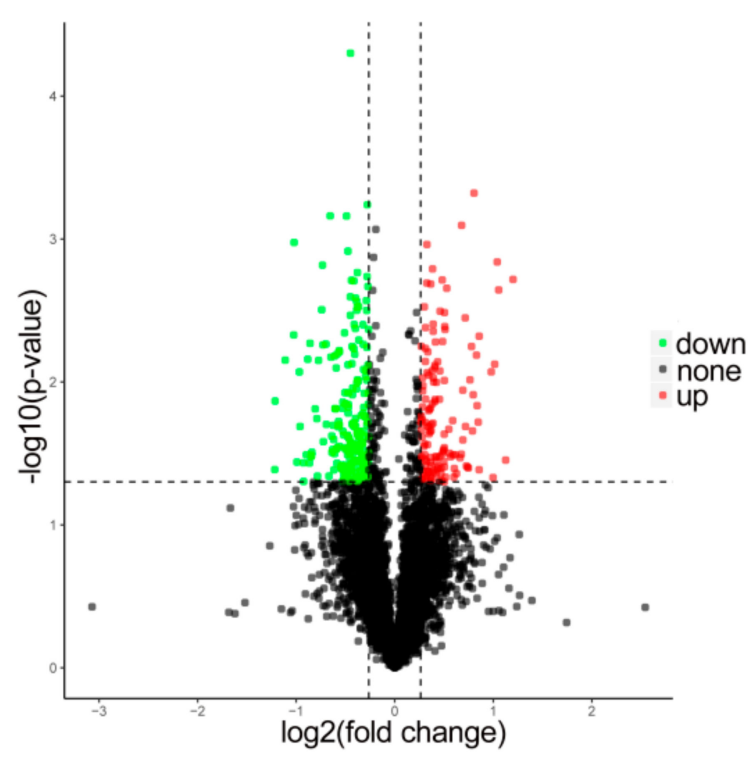

(a)

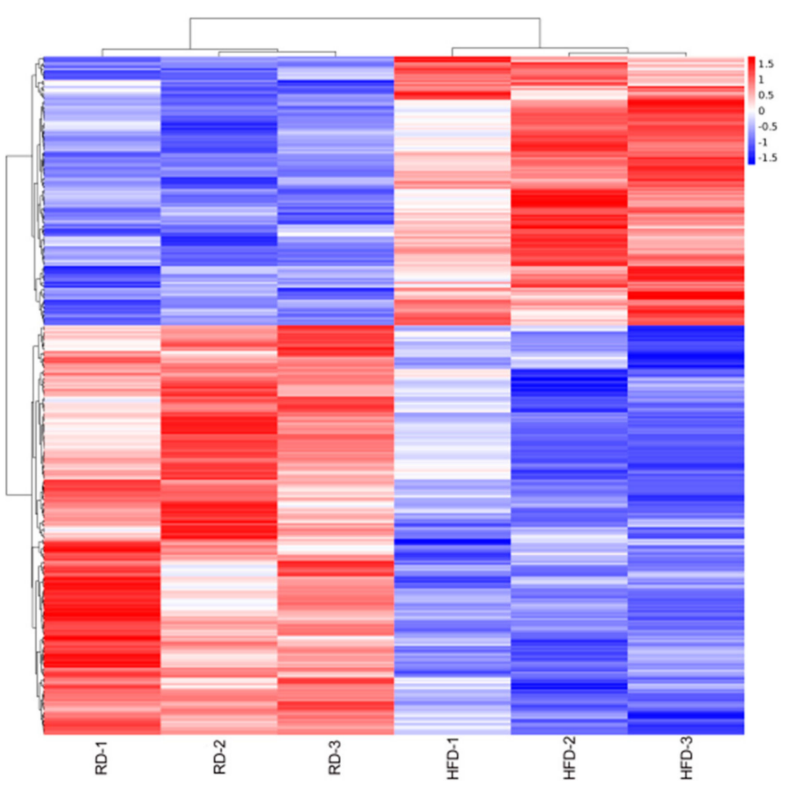

(b)

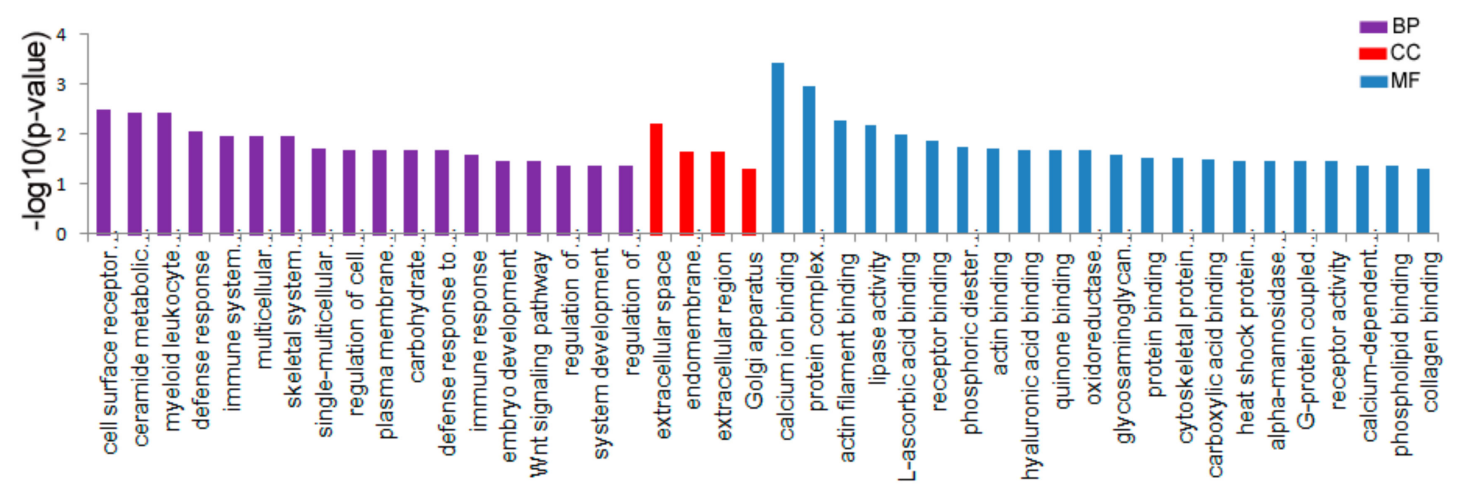

(c)

Figure 2. Identification and analysis of differentially expressed (DE) proteins. (a) Volcano map of DE proteins screened the HFD $(n=3)$ and RD $(n=3)$ groups and was built based on $\log 2$ (fold change) and -log10( $p$-value). (b) Hierarchical clustering analysis of the DE proteins in the samples of $\operatorname{HFD}(n=3)$ and $\operatorname{RD}(n=3)$ (red represents upregulated proteins, and blue represents downregulated proteins). (c) The results from the DE proteins were applied to a GO analysis, only showing the significantly enriched GO terms $(p<0.05)$. High-fat diet: HFD and restricted diet: RD.

\subsection{Identification and Analysis of DE Metabolites}

In our study, untargeted metabolomics based on UHPLC-MS/MS technology were used to measure the metabolite profiles of HFD and RD rabbits. The results of the PLSDA analysis showed clear segregation between the HFD and RD groups under both positive and negative modes, indicating a faithful representation of the data and good predictability (Figure 3a). Moreover, permutation tests validated that the PLS-DA model was no overfitting of data, revealing that dieting led to significant metabolic variations between the groups (Figure 3b).

After dietary restriction treatment, a total of $150 \mathrm{DE}$ metabolites were identified between the groups by the criteria of VIP $>1$ and fold changes $>1.5$ or fold changes $<0.667$ and $p<0.05$ (Table S4). Among these, 91 metabolites were upregulated, and 59 metabolites were downregulated (Figure 3c). When DE metabolites were compared with the Human Metabolome database (HMDB) database, 44 metabolites were annotated and classified as lipids and lipid-like molecules $(n=22)$, organic acids and derivatives $(n=6)$, organoheterocyclic compounds $(n=5)$, etc. (Table S5). Moreover, metabolites annotated with the LIPID MAPS database were mainly classified as fatty acyls (FTA; 
$n=10$ ), glycerophospholipids (GP; $n=13$ ), polyketides (PK; $n=2)$, and sterol lipids $(\mathrm{ST} ; n=6)$ (Table S6). The metabolite-metabolite interactions network showed that arachidonic acid (AA), L-dopa, cholecalciferol, hydrocortisone, and pyridoxamine are at the center of the network, indicating that these metabolites may play an important role in the process of dieting (Figure $3 \mathrm{~d}$ ).

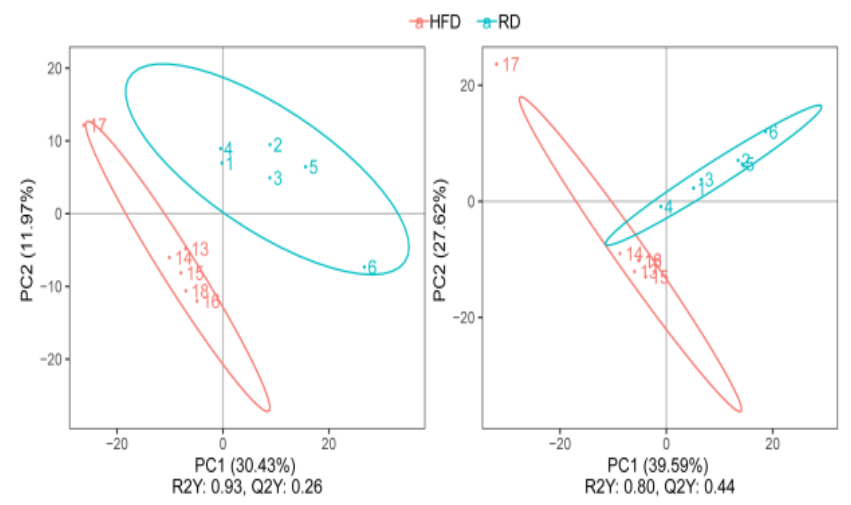

(a)

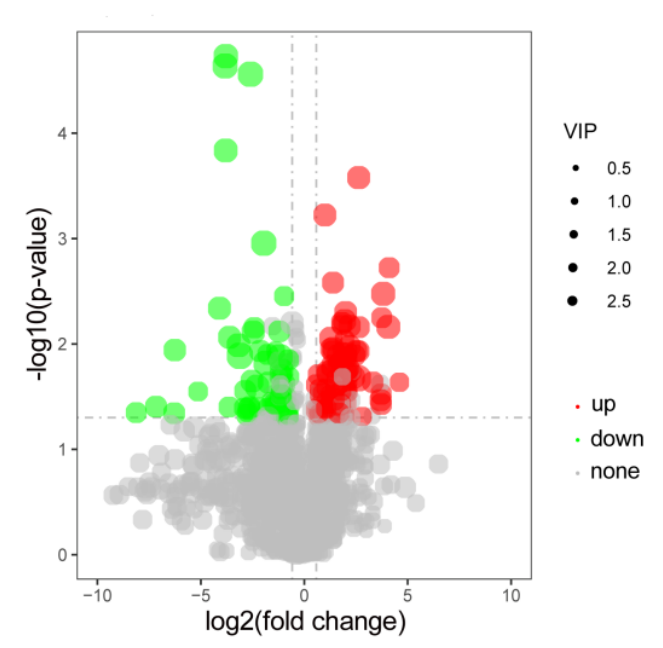

(c)

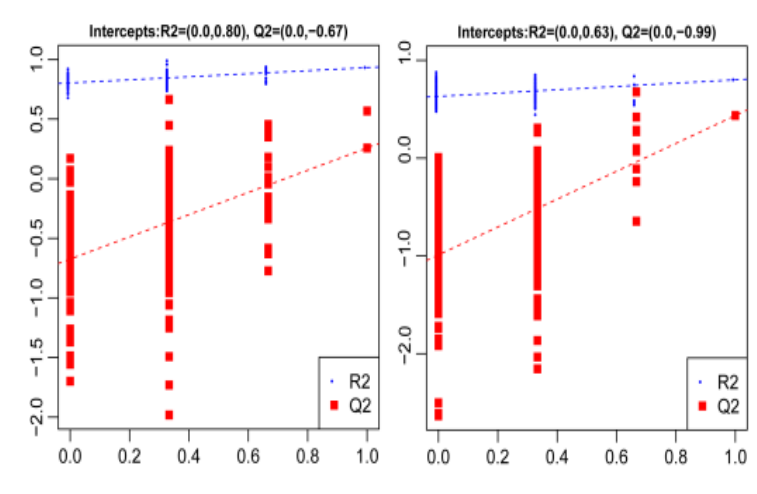

(b)

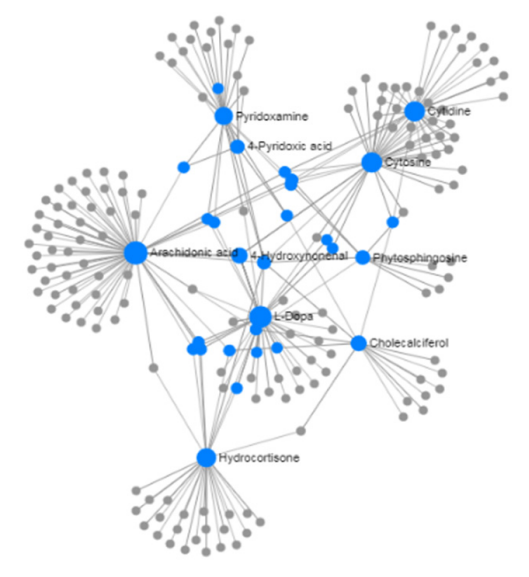

(d)

Figure 3. Identification and analysis of DE metabolites. (a) Partial least squares discriminant analysis (PLS-DA) of all metabolites in the samples of $\operatorname{HFD}(n=6)$ and $\operatorname{RD}(n=6)$ groups in the positive (left) and negative (right) ion modes. (b) The permutation test. (c) Volcano map of the DE metabolites screened between the HFD $(n=6)$ and RD $(n=6)$ groups and was built based on $\log 2$ (fold change) and $-\log 10(p$-value). (d) The metabolite-metabolite interaction network was built by using the MetaboAnalyst database. High-fat diet: HFD and restricted diet: RD.

\subsection{Integrated Analysis of Proteomics and Metabolomics}

To further identify the metabolic pathways impacted by dieting in obese rabbits, the MetaboAnalyst database was used to perform an integrative pathway analysis of the proteomics and metabolomics. According to the analysis results, some key pathways were considered to be associated with lipid metabolism, energy metabolism, vitamin metabolism, hormone metabolism, and amino acid metabolism and were significantly enriched $(p<0.05)$ after the dieting treatment, such as vitamin B6 metabolism, steroids metabolism, metabolism of lipids, and cholesterol metabolism, and fat digestion and absorption and free fatty acids regulate insulin secretion, etc. (Table S7 and Figure 4a). To better understand the changed pathways, the relationship network between the DE proteins and DE metabolites involved in significant pathways is illustrated in Figure 4b. Among these, mevalonic acid (MVA), AA, cholecalciferol, etc. were associated with multiple 
proteins, indicating that their interactions may play an important role in the regulation of dieting in obese rabbits.

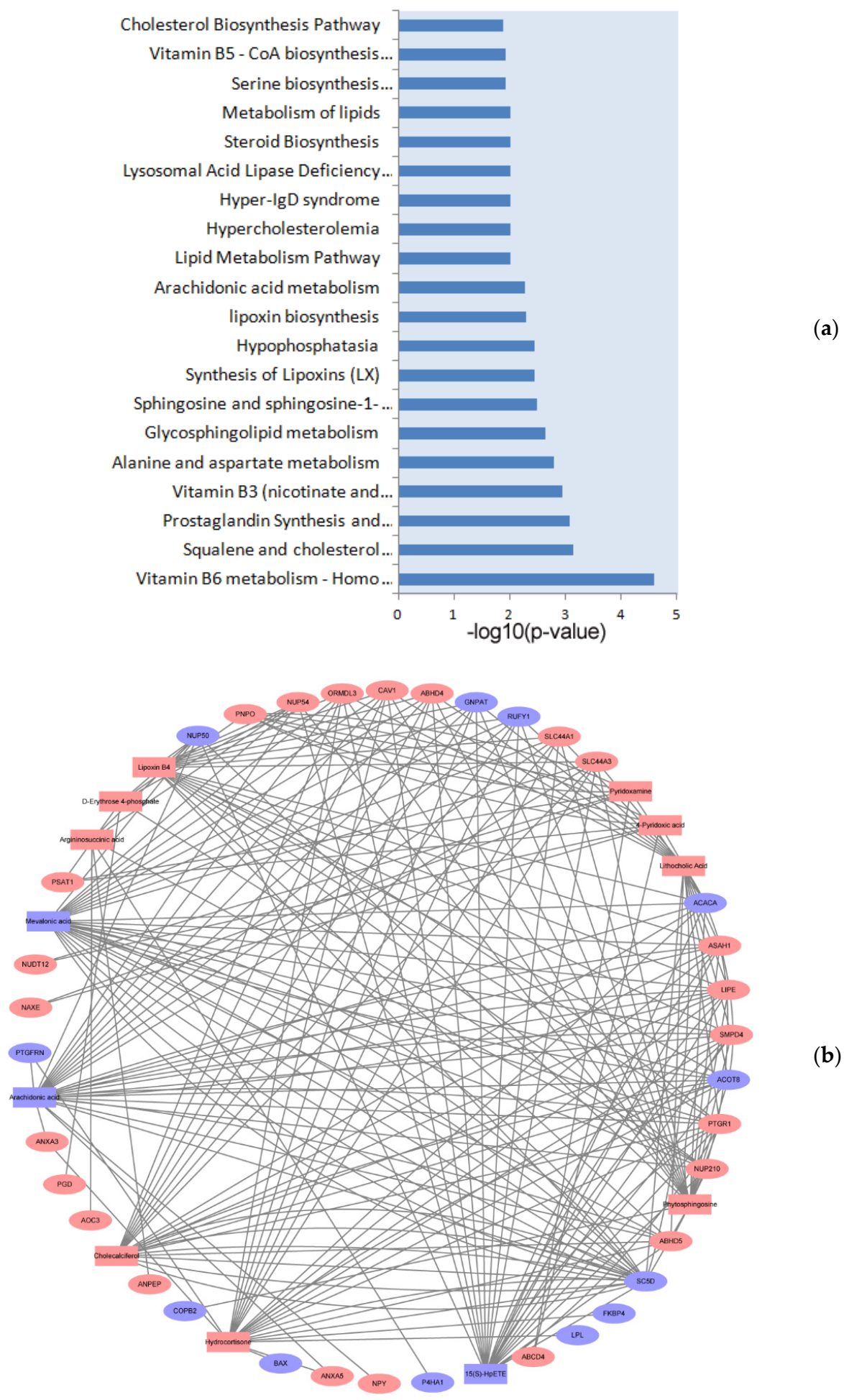

Figure 4. Integrated analysis of proteomics and metabolomics. (a) The integrated pathways analysis results of proteomics and metabolomics, only showing the top 20 pathways $(p<0.05)$. (b) We chose the pathways as an intermediate carrier, and the proteins and metabolites network were obtained. The circle represents proteins, and the triangle represents metabolites. Red represents the upregulated molecule, and blue represents the downregulated molecule. The line number indicates that proteins and metabolites are involved in multiple pathways. High-fat diet: HFD and restricted diet: RD. 


\section{Discussion}

Nowadays, the rapid epidemic of obesity poses a serious challenge to the prevention and treatment of chronic diseases throughout the world, including cardiovascular, diabetes, and some cancers [13]. The obesity epidemic has reached epidemic proportions not only in developed but, also, in developing countries. Thus, choosing a reasonable strategy to lose weight is important in tackling increasing obesity trends [14]. In our study, we established an obese rabbit model to investigate the molecular alterations underlying dieting by using an integrated proteomics and metabolomics analysis. However, only a limited number of proteins, metabolites, and pathways were discussed due to space limitations.

White adipose tissue (WAT) is a multi-depot organ that plays a regulatory role in energy homeostasis by adjusting the triglyceride (TG) storage [15]. Lipolysis and lipogenesis are key processes determining mature adipocyte sizes and mass. Here, when comparing the histomorphology between the HFD and RD rabbits, we found that RD caused adipocytes to appear smaller, indicating that lipolysis was increased or lipogenesis was decreased. In fact, the hydrolysis of fatty acids from TG stored in cellular lipid droplets is essential to ensure an adequate energy supply [16]. TG is gradually hydrolyzed by multifold lipases to fatty acids and glycerol and further to release into the blood for biological oxidation by multiple organs. In this process, adipocyte hormonal-sensitive lipase (HSL) is activated, and the TG stored in cellular lipid droplets is hydrolyzed, and the adipocyte volume is reduced [17]. Moreover, lipoprotein lipase (LPL) is also considered to be a key enzyme for lipid and lipoproteins metabolism [18]. The emerging data suggest the relative levels of LPL activity in adipose tissue determine whether lipids are partitioned towards storage or utilization and thereby lead to weight gain or loss [19]. During dieting, the LPL activity is decreased in adipose tissue and associated with an increase in the activity of the lipase [19,20]. HSL is known to hydrolyze TG, diglycerides (DG), cholesteryl esters, and retinyl esters, thereby providing the body with an energy substrate [21]. Such reciprocal regulation has probably evolved to maximize energy storage during periods of food availability and energy availability when food is scarce [19]. Acetyl-CoA carboxylase alpha (ACACA) is rate-limiting for the synthesis of long-chain fatty acids [22]. Our study showed that ACACA was lower in the RD group, suggesting that the body maintains an energy supply during starvation by hydrolyzing fat and inhibiting its synthesis. Moreover, the proteomic analysis also indicated an upregulation of the sphingolipid metabolism pathway ( $\mathrm{N}$-acylsphingosine amidohydrolase 1 and sphingomyelin phosphodiesterase 4) and adipocytokine signaling pathway (CD36 molecule, neuropeptide Y, and phosphoenolpyruvate carboxykinase 1) in the $\mathrm{RD}$ group. Sphingolipid biosynthesis is required for adipocyte cell viability and normal metabolic function [23]. The dysregulation of the adipocytokine signaling pathway is related to eating disorders and may be the common pathway for body weight regulation in complex diseases related to unhealthy lifestyles [24]. Together, these findings suggest that $\mathrm{RD}$ leads to a decrease in adipocyte sizes and a series of disorders in the lipid metabolism in female obese rabbits. However, whether resuming a normal or HFD after dieting will restore obesity needs further study.

Lipolysis is a dynamic process involving an elaborate network of allosteric regulators, hormones, signaling pathways, and transcription factors, etc. Among these 343 DE proteins, some proteins were reported to be associated with amino acid metabolism, suggesting the dysregulation of amino acid in obese rabbits after treatment with a RD. Amine oxidase copper containing 3 (AOC3), phosphoglycerate dehydrogenase (PHGDH), and phosphoserine aminotransferase 1 (PSAT1) were upregulated in the glycine, serine, and threonine metabolism pathways, and extensive work revealed that these pathways are key players in the antibacterial ability $[25,26]$. AOC 3 expressed by adipocytes catalyzes the oxidative deamination of amines to release $\mathrm{H}_{2} \mathrm{O}_{2}$, ammonia, and aldehyde [27]. With the finding about the importance of $\mathrm{H}_{2} \mathrm{O}_{2}$ in the insulin signaling pathway, a possible role of AOC3 in adipocytes may be found [28]. PHGDH and PSAT1 are necessary enzymes participating in serine synthesis $[29,30]$. However, both PHGDH and PSAT1 are regulators and associated with an increased risk for multiple cancer progression and metastasis, indicating a possible 
link between dieting and cancers [31,32]. Moreover, we also observed that some amino acid metabolism-related proteins were dysregulated, such as ubiquitin-specific peptidase 15 (USP15), ubiquitin family domain containing 1 (UBFD1), procollagen c-endopeptidase enhancer (PCOLCE), and alanyl aminopeptidase (ANPEP). Our results are consistent with the research on mental diseases in the rat model [10]. However, the specificity of these changes relative to other diseases requires further work to measure.

The metabolomics of obese rabbits treated with a RD showed changes of metabolites that were part of the phospholipid metabolism (phosphatidylcholine (PC), phosphatidylethanolamine (PE), diacylglycerol (DAG), phosphatidic acid (PA), and lysophosphatidic acid (LPA)) and fatty acid metabolism (arachidonic acid (AA)). Adipocytes function depends on the homeostasis of important cellular lipid mediators and lipid structural components of the biological membranes required for accurate functional responses [33]. After the HFD treatment, adipocytes initiate a series of membrane lipid remodeling in response to excessive energy intake. These changes in adipocytes may lead to a change in membrane composition, thereby resulting in changes in membrane fluidity and cellular physiology [34]. After RD treatment, we found that lower levels of most PC were expressed, but PE was increased in the RD group. The dynamic changes of PC and PE are associated with the structure and function of the membrane and could represent a compensatory response aimed at resisting the fluidity change [35,36]. Moreover, DAG, PA, LPA, and AA were decreased similarly in the RD group. These molecules are key contributors to membrane lipid remodeling and fluidity [33,37]. Interestingly, as an important molecule, PC is a major source of DAG, PA, LPA, and AA [38]. Various studies have pointed out the importance of DAG, PA, LPA, and AA for signal transmissions, which are pivotal second messengers controlling numerous cellular responses and can be further metabolized to other signaling molecules $[39,40]$. The data of proteomics also found that some proteins located in the membrane were significantly altered, which mainly included adhesion $G$ protein-coupled receptor E5, aquaporin 4, frizzled class receptor 4, LDL receptor-related protein $1 \mathrm{~B}$, mannose receptor $C$ type 2 , notch receptor 2, Oryctolagus cuniculus very low-density lipoprotein receptor, annexin A3, annexin A5, and annexin A7. These proteins, except for annexin A3, annexin A5, and annexin A7, were downregulated, which suggests a deficit of signal transduction-related proteins in the RD group. Our study provided the possibility for a positive role of annexin in lipolysis. Increasing evidence revealed that the activities of annexin were facilitated through their diverse interactions with a plethora of lipids and proteins [41]. The annexin family are active in adipose tissue, and annexin A1, annexin A6, and annexin A3 have been linked to the control of adipocyte lipolysis and adiponectin release [41,42]. However, their functions in adipose tissue are less well-measured. Moreover, the downregulation of cytoskeleton-related proteins (cytoskeleton-associated protein 4, microtubule actin crosslinking factor 1, microtubule-associated scaffold protein 1, tubulin folding cofactor $\mathrm{B}$, tubulin gamma complex-associated protein 3, and the fibronectin type III domain containing 3A) in the RD group suggested changes of the dynamics of the cytoskeleton [43]. The alterations of the cell shapes are accompanied by changes in the cytoskeletal organization and contacts with fibronectin, actin, and several cytoskeletal proteins [44]. Thus, we concluded that dieting results in membrane and cytoskeleton remodeling, along with a series of alterations in membrane composition and biophysical properties in the adipocytes.

Furthermore, a network analysis based on the pathway revealed that several identified nodes were notable, such as the reduction of MVA, AA, 15(S)-HpETE, farnesyl-diphosphate farnesyltransferase 1 (FDFT1), and sterol-C5-desaturase (SC5D) and enhancement of cholecalciferol, hydrocortisone, lipoxin B4, and lithocholic acid in the RD group. Given that these molecules strongly changed in multiple pathways, it is implied that they may play complex functions in regulating the metabolism or diseases impacted by dieting. It is expected that MVA was decreased because cholesterol synthesis has been associated with the mevalonic acid pathway [45]. Mevalonate metabolites affect not only transcriptional events but, also, 
post-transcriptional events that, in turn, impact various biological activities, including the energy metabolism [46]. The dysregulation of SC5D and FDFT1 may be responsible for the cholesterol-related molecular alterations, because they are the key roles involved in the processes of cholesterol synthesis and metabolism [47,48]. However, the discovery of decreased 15(S)-HpETE in the RD group was novel. 15(S)-HpETE is the product of AA formed in the 15-lipoxygenase pathway [49]. In adipose tissue, it is thought to have a causal relationship to antiangiogenics [50]. Extensive work revealed that inflammation and angiogenesis are interdependent [51]. Inflammatory cells participate in the angiogenic process by secreting proinflammatory and anti-inflammatory cytokines that can control endothelial cell proliferation, survival, and apoptosis, as well as their migration and activation, which are critical events in angiogenesis [52]. Due to the codependency of inflammation and angiogenesis, suppression of the angiogenic response may suppress inflammation [49]. Interestingly, cholecalciferol, hydrocortisone, lipoxin B4, and lithocholic acid were upregulated, which are known to counteract inflammation responses [53-56]. Moreover, a pathways analysis indicated that the above molecules have effects on many pathways, including sphingosine and sphingosine-1-phosphate metabolism, the metabolism of lipids, the short of Lipoxin, ovarian steroidogenesis, etc. In addition to the above molecular changes, the levels of branched fatty acid esters of hydroxy fatty acids (FAHFA) were markedly increased. Our work supports the early study that lipolysis in adipocytes is associated with marked increases in the FAHFA levels [57]. PAHFA is a novel class of endogenous lipids that also shows a great impact on lipid inflammation in the obese population [58]. Thus, we speculated that dieting regulated the expression of anti-inflammatory factors, thereby mitigating the inflammation induced by HFD. However, this regulatory mechanism has not been fully studied.

Taken together, we applied an integrated proteomics and metabolomics analysis to investigate the effects of dieting on the proteins and metabolites in obese rabbits. We found that metabolomics and proteomics were changed markedly after rabbits treated with a $\mathrm{RD}$, and the amino acid metabolism, lipid metabolism, and membrane and cytoskeleton reconstruction may be the key processes during dieting. Moreover, the body may change the expression of anti-inflammatory factors to fight inflammation induced by HFD. Our study provides further insights into the research of dieting and may contribute to the prevention and treatment of obesity. However, multiple limitations exist in this study. For example, given that age influences the metabolic rates associated with proteins and metabolites metabolism, the rabbit age is a critical factor that should be taken seriously. Since the specimens were animals, the number of specimens was relatively small. Moreover, we did not use a different method or database to verify the analysis results. One method cannot cover every aspect of the real physical changes. Thus, the functional verification of these DE proteins and DE metabolites and their network will be important to consider in the future.

Supplementary Materials: The following are available online at https://www.mdpi.com/article/ 10.3390/biology10040321/s1: Table S1: The list of all identified proteins. Table S2: The list of DE proteins between the HFD and RD groups. Table S3: The GO analysis results of the DE proteins. Table S4: The list of DE metabolites between the HFD and RD groups. Table S5: The annotation results when the DE metabolites were compared with the HMDB database. Table S6: The annotation results when the DE metabolites were compared with the LIPID MAPS database. Table S7: Integrated pathway analysis of the proteomics and metabolomics.

Author Contributions: J.W., X.J. and S.L. conceived and designed the study; T.T. and Y.L. collected data and conducted the research; and T.P. and J.S. wrote the paper. All authors have read and agreed to the published version of the manuscript.

Funding: Our work was funded by the China Agricultural Research System (grant no. CARS-44-A-2).

Institutional Review Board Statement: All experimental protocols were performed under the direction of the Institutional Animal Care and Use Committee from the College of Animal Science and Technology, Sichuan Agricultural University, China (DKY-B2019202015). 
Informed Consent Statement: Not applicable.

Data Availability Statement: All data generated or analyzed during this study are included.

Conflicts of Interest: The authors declare no conflict of interest.

\section{References}

1. Popkin, B.M.; Doak, C.M. The Obesity Epidemic Is a Worldwide Phenomenon. Nutr. Rev. 2010, 56, 106-114. [CrossRef]

2. Trayhurn, P.; Beattie, J.H. Physiological role of adipose tissue: White adipose tissue as an endocrine and secretory organ. Proc. Nutr. Soc. 2001, 60, 329-339. [CrossRef]

3. Llewellyn, C.; Wardle, J. Behavioral susceptibility to obesity: Gene-Environment interplay in the development of weight. Physiol. Behav. 2015, 152, 494-501. [CrossRef]

4. Swinburn, B.; Sacks, G.; Hall, K. The global obesity pandemic: Shaped by global drivers and local environments. Lancet 2011, 378, 804-818. [CrossRef]

5. Branco, J.C.; Motta, J.; Wiener, C.; Oses, J.P.; Pedrotti Moreira, F.; Spessato, B.; Dias, L.; Da Silva, R. Association between obesity and suicide in woman, but not in man: A population-based study of young adults. Psychol. Health Med. 2016, 22, 275-281. [CrossRef]

6. Brugman, E.; Meulmeester, J.F.; Spee-Van, D.W.A.; Beuker, R.J.; Zaadstra, B.M.; Radder, J.J.; Verloove-Vanhorick, P.S. Dieting, weight and health in adolescents in The Netherlands. Int. J. Obes. Relat. Metab. Disord. J. Int. Assoc. Study Obes. 1997, 21, 54-60. [CrossRef]

7. French, S.A.; Jeffery, R.W. Consequences of dieting to lose weight: Effects on physical and mental health. Health Psychol. 1994, 13, 195-212. [CrossRef]

8. Kemps, E.; Tiggemann, M.; Marshall, K. Relationship between dieting to lose weight and the functioning of the central executive. Appetite. 2005, 45, 287-294. [CrossRef] [PubMed]

9. Cogan, J.C.; Ernsberger, P. Dieting, Weight, and Health: Reconceptualizing Research and Policy. J. Soc. Issues. 2010, 55, 187-205. [CrossRef]

10. Zhang, Y.; Yuan, S.; Pu, J.; Yang, L.; Xie, P. Integrated Metabolomics and Proteomics Analysis of Hippocampus in a Rat Model of Depression. Neuroence. 2017, 371, 207-220. [CrossRef] [PubMed]

11. Shao, J.; Wang, J.; Li, Y.; Elzo, M.A.; Tang, T.; Lai, T.; Ma, Y.; Gan, M.; Wang, L.; Jia, X.; et al. Growth, behavioural, serum biochemical and morphological changes in female rabbits fed high-fat diet \%J Journal of Animal Physiology \& Animal Nutrition. J. Anim. Physiol. Anim. Nutr. 2020, 105, 345-353.

12. Tang, H.; Zhang, X.; Gong, B.; Yan, Y.; Shi, Q. Proteomics and metabolomics analysis of tomato fruit at different maturity stages and under salt treatment. Food Chem. 2020, 311, 126009.126001-126009.126010. [CrossRef]

13. James, P.T.; Neville, R.; Rachel, L. The obesity epidemic, metabolic syndrome and future prevention strategies. Eur. J. Cardiovasc. Prev. 2019, 11, 3-8. [CrossRef] [PubMed]

14. Molarius, A.; Lindén Ostrm, M.; Karlsson, J. Desire to lose weight and need of weight loss support in the adult population Results from a cross: Ectional study in Sweden. Obes. Ence Pract. 2020, 6, 373-381. [CrossRef]

15. Trayhurn, P.; Wood, I.S. Adipokines: Inflammation and the pleiotropic role of white adipose tissue. Br. J. Nutr. 2004, 92, 347-355. [CrossRef] [PubMed]

16. Large, V.; Peroni, O.; Letexier, D.; Ray, H.; Beylot, M. Metabolism of lipids in human white adipocyte. Diabetes Metab. 2004, 30 , 294-309. [CrossRef]

17. Mezghanni, N.; Chaabouni, K.; Chtourou, H.; Masmoudi, L.; Mejdoub, H. Effect of exercise training intensity on body composition, lipid profile, and insulin resistance in young obese women. Afr. J. Microbiol. Res. 2012, 6, 2481-2488.

18. Eckel, R.H. Lipoprotein lipase. A multifunctional enzyme relevant to common metabolic diseases. N. Engl. J. Med. 1989, 320, 1060. [PubMed]

19. Mead, J.R.; Irvine, S.A.; Ramji, D.P. Lipoprotein lipase: Structure, function, regulation, and role in disease. J. Mol. Med. 2002, 80, 753-769. [CrossRef] [PubMed]

20. Taskinen, M.-R.; Nikkilä, E.A. Basal and postprandial lipoprotein lipase activity in adipose tissue during caloric restriction and refeeding. Metabolism 1987, 36, 625-630. [CrossRef]

21. Guenter, H.; Robert, Z.; Marianne, H.; Christian, T.; Georg, W.; Elke, W.; Wolfgang, S.; Thomas, M.M.; Erwin, F.W.; Rudolf, Z. Hormone-sensitive lipase deficiency in mice causes diglyceride accumulation in adipose tissue, muscle, and testis. J. Biol. Chem. 2002, 277, 4806-4815.

22. Mao, J.; Marcos, S.; Davis, S.K.; Burzlaff, J.; Seyfert, H.M. Genomic distribution of three promoters of the bovine gene encoding acetyl-CoA carboxylase alpha and evidence that the nutritionally regulated promoter I contains a repressive element different from that in rat. Biochem. J. 2001, 358, 127-135. [CrossRef]

23. Alexaki, A.; Clarke, B.A.; Gavrilova, O.; Ma, Y.; Zhu, H.; Ma, X.; Xu, L.; Tuymetova, G.; Larman, B.C.; Allende, M.L. De Novo Sphingolipid Biosynthesis Is Required for Adipocyte Survival and Metabolic Homeostasis. J. Biol. Chem. 2017, 292, 3929-3939. [CrossRef]

24. Min, Z.; Xiaomo, L.I.; Hong, Q.U. EDdb: A web resource for eating disorder and its application to identify an extended adipocytokine signaling pathway related to eating disorder. Sci. China. Life Sci. 2013, 56, 1086-1096. 
25. Wu, C.-W.; Zhao, X.-L.; Wu, X.-J.; Wen, C.; Li, H.; Chen, X.-H.; Peng, X.-X. Exogenous glycine and serine promote growth and antifungal activity of Penicillium citrinum W1 from the south-west Indian Ocean. FEMS Microbiol. Lett. 2015, 362, fnv040.

26. Zhao, X.; Liu, Z.; Liu, Z.; Meng, R.; Shi, C.; Chen, X.; Bu, X.; Guo, N. Phenotype and RNA-seq-Based transcriptome profiling of Staphylococcus aureus biofilms in response to tea tree oil. Microb. Pathog. 2018, 123, 304-313.

27. Salter-Cid, L.M. Anti-Inflammatory Effects of Inhibiting the Amine Oxidase Activity of Semicarbazide-Sensitive Amine Oxidase. J. Pharmacol. Exp. Ther. 2005, 315, 553-562. [CrossRef]

28. Henriksen, E.J. Effects of $\mathrm{H} 2 \mathrm{O} 2$ on insulin signaling the glucose transport system in mammalian skeletal muscle. Methods Enzymol. 2013, 528, 269-278. [PubMed]

29. Shimizu, M.; Furuya, S.; Shinoda, Y.; Mitoma, J.; Okamura, T.; Miyoshi, I.; Kasai, N.; Hirabayashi, Y.; Suzuki, Y. Functional analysis of mouse 3-phosphoglycerate dehydrogenase (Phgdh) gene promoter in developing brain. J. Neurosci. Res. 2004, 76, 623-632. [CrossRef] [PubMed]

30. Yu, J.; Xiao, F.; Guo, Y.; Deng, J.; Liu, B.; Zhang, Q.; Li, K.; Wang, C.; Chen, S.; Guo, F. Hepatic Phosphoserine Aminotransferase 1 Regulates Insulin Sensitivity in Mice via Tribbles Homolog 3. Diabetes. 2015, 64, 1591-1602. [CrossRef] [PubMed]

31. Gao, S.; Ge, A.; Xu, S.; You, Z.; Ning, S.; Zhao, Y.; Pang, D. PSAT1 is regulated byATF4 and enhances cell proliferation via the GSK3 $\beta$ / $\beta$-catenin/cyclin D1 signaling pathway in ER-negative breast cancer. J. Exp. Clin. Cancer Res. 2017, 36, 179. [CrossRef]

32. Huang, S.-P.; Chan, Y.-C.; Yu, S.; Lin, Y.-F. Overexpression of PSAT1 Gene is a Favorable Prognostic Marker in Lower-Grade Gliomas and Predicts a Favorable Outcome in Patients with IDH1 Mutations and Chromosome 1p19q Codeletion. Cancers 2019, 12, 13. [CrossRef]

33. Pietilainen, K.H.; Róg, T.; Seppanen-Laakso, T.; Virtue, S.; Gopalacharyulu, P.; Tang, J.; Rodriguez-Cuenca, S.; Maciejewski, A.; Naukkarinen, J. Association of Lipidome Remodeling in the Adipocyte Membrane with Acquired Obesity in Humans. PLoS Biol. 2011, 9, 295-304. [CrossRef]

34. Niemel, P.S.; Ollila, S.; Hyv Nen, M.T.; Karttunen, M.; Vattulainen, I. Assessing the Nature of Lipid Raft Membranes. Plos Comput. Biol. 2007, 3, 304-312. [CrossRef] [PubMed]

35. Mysore, R.; Liebisch, G.; Zhou, Y.; Olkkonen, V.M. Angiopoietin-like 8 (Angptl8) controls adipocyte lipolysis and phospholipid composition. Chem. Phys. Lipids 2017, 207, 246-252. [CrossRef] [PubMed]

36. Barsukov, L.I.; Bergelson, L.D.; Spiess, M.; Hauser, H.; Semenza, G. Phospholipid topology and flip-flop in intestinal brush-border membrane. Biochim. Biophys. Acta 2019, 862, 87-99. [CrossRef]

37. Larijani, B.; Dufourc, E.J. Polyunsaturated phosphatidylinositol and diacylglycerol substantially modify the fluidity and polymorphism of biomembranes: A solid-state deuterium NMR study. Lipids 2006, 41, 925-932. [CrossRef] [PubMed]

38. Gibellini, F.; Smith, T.K. The Kennedy pathway—Synthesis of phosphatidylethanolamine and phosphatidylcholine. Iubmb Life. 2010, 62, 414-428. [CrossRef] [PubMed]

39. Brose, N.; Betz, A.; Wegmeyer, H. Divergent and convergent signaling by the diacylglycerol second messenger pathway in mammals. Curr. Opin. Neurobiol. 2004, 14, 328-340. [CrossRef]

40. Yang, L.; Andrews, D.A.; Low, P.S. Lysophosphatidic acid opens a Ca++ channel in human erythrocytes. Blood 2000, 95, $2420-2425$. [CrossRef]

41. Grewal, T.; Enrich, C.; Rentero, C.; Buechler, C. Annexins in Adipose Tissue: Novel Players in Obesity. Int. J. Mol. Sci. 2019, 20, 3449. [CrossRef]

42. Watanabe, T.; Ito, Y.; Sato, A.; Hosono, T.; Niimi, S. Annexin A3 as a negative regulator of adipocyte differentiation. J. Biochem. 2012, 152, 355-363. [CrossRef]

43. Nobusue, H.; Onishi, N.; Shimizu, T.; Sugihara, E.; Oki, Y.; Sumikawa, Y.; Chiyoda, T.; Akashi, K.; Saya, H.; Kano, K. Regulation of MKL1 via actin cytoskeleton dynamics drives adipocyte differentiation. Nat. Commun. 2014, 5, 3368. [CrossRef] [PubMed]

44. Kawaguchi, N. ADAM12 induces actin cytoskeleton and extracellular matrix reorganization during early adipocyte differentiation by regulating 1 integrin function. J. Cell Sci. 2003, 116, 3893-3904. [CrossRef] [PubMed]

45. Liu, X.; You, W.; Cheng, H.; Zhang, Q.; Song, E. Effect of mevalonic acid on cholesterol synthesis in bovine intramuscular and subcutaneous adipocytes. J. Appl. Genet. 2016, 57, 113-118. [CrossRef]

46. Goto, T.; Nagai, H.; Egawa, K.; Kim, Y.I.; Kato, S.; Taimatsu, A.; Sakamoto, T.; Ebisu, S.; Hohsaka, T.; Miyagawa, H.J.B.J. Farnesyl pyrophosphate regulates adipocyte functions as an endogenous PPAR $\gamma$ agonist. Biochem. J. 2011, 438, 111-119. [CrossRef]

47. Nishi, S.; Nishino, H.; Ishibashi, T. cDNA cloning of the mammalian sterol C5-desaturase and the expression in yeast mutant. Biochim. Biophys. Acta Gene Struct. Expr. 2000, 1492, 211. [CrossRef]

48. Mori, M.; Li, G.; Abe, I.; Nakayama, J.; Guo, Z.; Sawashita, J.; Ugawa, T.; Nishizono, S.; Serikawa, T.; Higuchi, K. Lanosterol synthase mutations cause cholesterol deficiency-associated cataracts in the Shumiya cataract rat. J. Clin. Investig. 2006, 116, 395-404. [CrossRef]

49. Soumya, S.J.; Binu, S.; Helen, A.; Kumar, K.A.; Reddanna, P.; Sudhakaran, P.R. Effect of 15-lipoxygenase metabolites on angiogenesis: 15(S)-HPETE is angiostatic and 15(S)-HETE is angiogenic. Inflamm. Res. 2012, 61, 707-718. [CrossRef]

50. Soumya, S.J.; Binu, S.; Helen, A.; Reddanna, P.; Sudhakaran, P.R. 15-LOX metabolites and angiogenesis: Angiostatic effect of 15(S)-HPETE involves induction of apoptosis in adipose endothelial cells. Peer] 2014, 2, e635. [CrossRef] [PubMed]

51. Clavel, G.; Valvason, C.; Yamaoka, K.; Lemeiter, D.; Bessis, N. Relationship between angiogenesis and inflammation in experimental arthritis. Eur. Cytokine Netw. 2006, 17, 202-210. [PubMed] 
52. Naldini, A.; Carraro, F. Role of inflammatory mediators in angiogenesis. Curr. Drug Targets Inflamm Allergy 2005, 4, 3-8. [CrossRef] [PubMed]

53. Matias, P.J.; Jorge, C.; Ferreira, C.; Borges, M.; Aires, I.; Amaral, T.; Gil, C.; Cortez, J.; Ferreira, A. Cholecalciferol supplementation in hemodialysis patients: Effects on mineral metabolism, inflammation, and cardiac dimension parameters. Clin. J. Am. Soc. Nephrol. 2010, 5, 905. [CrossRef] [PubMed]

54. Schleimer, R.P. Potential regulation of inflammation in the lung by local metabolism of hydrocortisone. Am. J. Respir. Cell Mol. Biol. 2012, 4, 166-173. [CrossRef] [PubMed]

55. Karra, L.; Haworth, O.; Priluck, R.; Levy, B.D.; Levi-Schaffer, F. Lipoxin BLipoxin B-4 promotes the resolution of allergic inflammation in the upper and lower airways of mice. Mucosal Immunol. 2015, 8, 852-862. [CrossRef]

56. Moretti, A.; Li, Q.; Chmielowski, R.; Joseph, L.; Moghe, P.; Uhrich, K. Nanotherapeutics Containing Lithocholic Acid-Based Amphiphilic Scorpion-Like Macromolecules Reduce In Vitro Inflammation in Macrophages: Implications for Atherosclerosis. Nanomaterials 2018, 8, 84. [CrossRef]

57. Tan, D.; Ertunc, M.E.; Konduri, S.; Zhang, J. Discovery of FAHFA-containing Triacylglycerols and Their Metabolic Regulation. J. Am. Chem. Soc. 2019, 141, 8798-8806. [CrossRef] [PubMed]

58. Zhang, T.; Chen, S.; Syed, I.; St Hlman, M.; Kolar, M.J.; Homan, E.A.; Chu, Q.; Smith, U.; Borén, J.; Kahn, B.B. A LC-MS-based workflow for measurement of branched fatty acid esters of hydroxy fatty acids. Nat. Protoc. 2016, 11, 747. [CrossRef] 